\title{
Variabilidade espacial do pH, teores de matéria orgânica e micronutrientes em profundidades de amostragem num Latossolo Vermelho sob semeadura direta
}

\author{
Space variability of the $\mathrm{pH}$ values, contents of organic matter and micronutrients in samplings \\ depth in a Acrustox under no-till system
}

\author{
Luiz Antônio Zanão Júnior ${ }^{\mathrm{I}}$ Regina Maria Quintão Lana" ${ }^{\mathrm{II}}$ Ednaldo Carvalho Guimarães ${ }^{\mathrm{III}}$
}

\section{RESUMO}

A variabilidade espacial dos índices de fertilidade do solo aumenta com a semeadura direta, constatando-se diferenças nos teores de nutrientes de uma camada superficial do solo em relação à outra logo abaixo. Disto, resulta a necessidade de definir novos procedimentos de amostragem para contemplar essas alterações. Com o objetivo de avaliar a variabilidade espacial do $\mathrm{pH}$ em água, teores de matéria orgânica e os micronutrientes boro $(\mathrm{B})$, cobre $(\mathrm{Cu})$, ferro $(\mathrm{Fe})$, manganês $(\mathrm{Mn})$ e zinco $(\mathrm{Zn})$, desenvolveu-se este trabalho num Latossolo Vermelho distrófico, textura muito argilosa, cultivado desde 1995 sob semeadura direta, em Uberlândia MG. A coleta das amostras do solo foi realizada em intervalos regulares de $50 \mathrm{~m}$, em malha regular de 121 pontos, em três profundidades $(0-10 \mathrm{~cm}, 10-20 \mathrm{~cm}$ e $0-20 \mathrm{~cm})$. Os dados foram avaliados por estatística descritiva e geoestatística, com base no ajuste de semivariogramas. Maiores teores de matéria orgânica, Mn e $\mathrm{Zn}$ foram verificados nos primeiros $10 \mathrm{~cm}$ do solo, enquanto que, para $\mathrm{Fe}$, os maiores teores ocorreram na camada de $10-20 \mathrm{~cm}$. As maiores variabilidades horizontais foram encontradas para $B$ e as menores para $\mathrm{pH}$ em água. Para os atributos que apresentaram dependência espacial, esta foi classificada como moderada ou forte. A maioria dos dados se ajustou ao semivariograma de modelo exponencial, seguido do esférico e do linear. Apenas a variável zinco, na profundidade de $0-20 \mathrm{~cm}$, não apresentou dependência espacial. Os resultados obtidos devem ser considerados na definição de planos de amostragem do solo e no manejo da adubação da área em questão, através da análise do alcance e das variabilidades horizontal e vertical dos atributos químicos estudados.

Palavras-chave: geoestatística, fertilidade do solo, semivariograma, amostragem.

\begin{abstract}
The space variability of the indexes of fertility of the soil increases with the no-till system, being verified differences in the nutrients contents of a superficial layer of the soil in relation to the other lower layer. Therefore, it requires the definition of new sampling procedures to contemplate those alterations. The study was aimed at evaluating the spatial variability of the $\mathrm{pH}$, organic matter and micronutrients $(B$, $\mathrm{Cu}, \mathrm{Fe}, \mathrm{Mn}$ and $\mathrm{Zn}$ ) in a Acrustox loamy texture, cultivated since 1995 under no till system. The soil was sampled in regular intervals of $50 \mathrm{~m}$, in regular mesh of 121 points, in three depths $(0-10 \mathrm{~cm}, 10-20 \mathrm{~cm}$ and $0-20 \mathrm{~cm})$. The data evaluated by descriptive statistics and geostatistics, based on semivariograms adjustment. Larger contents of organic matter, $\mathrm{Mn}$ and $\mathrm{Zn}$ were verified in the first $10 \mathrm{~cm}$ of the soil, whereas contrary behaviors were observed for Fe. The highest variabilities were found for $B$ and the lowest for $\mathrm{pH}$ in water. For the attributes that presented spatial dependence, this was classified as moderate or strong. Most of the data was adjusted to the semivariograms of exponential model, folloed by the spherical model. Only the variable zinc, in the depth of $0-20 \mathrm{~cm}$, did not present spatial dependence. The results obtained results should be considered in the definition of plans of soil sampling and management fertilization of the studied area by the analysis of range and horizontal and vertical variability of the chemical attributes evaluated.
\end{abstract}

Key words: geostatístics, soil fertility, semivariogram, sampling.

\section{INTRODUÇÃO}

O estudo da variabilidade espacial dos nutrientes no solo é uma etapa importante na agricultura

IPrograma de Pós-graduação em Solos e Nutrição de Plantas, Departamento de Solos, Universidade Federal de Viçosa (UFV). Av. P.H. Rolfs, s/n, Centro, 36570-000, Viçosa, MG, Brasil. E-mail: luizantoniozanao@yahoo.com. Autor para correspondência. IIInstituto de Ciências Agrárias, Universidade Federal de Uberlândia (UFU), Uberlândia, MG, Brasil. E-mail: rmqlana@iciag.ufu.br. IIIFaculdade de Matemática, UFU, Uberlândia, MG, Brasil. E-mail: ecg@ufu.br. 
de precisão. Nesta fase, a utilização dos semivariogramas permite planejar desenhos ótimos de amostragem do solo, no intuito de capturar a variabilidade em escalas espaciais.

A variabilidade espacial dos nutrientes pode não ser igual entre si, ou seja, alguns nutrientes necessitam de números maiores de amostras que outros, para que se possa conhecer o seu comportamento em relação a uma determinada área. Uma maneira de solucionar estes problemas seria a adoção de métodos geoestatísticos para avaliar a variabilidade espacial da fertilidade do solo (DEUTSCH \& JOURNEL, 1998). A variabilidade do solo é uma conseqüência de complexas interações dos fatores e processos de sua formação, sendo influenciada pelas práticas de manejo e pelas culturas. Áreas pedologicamente similares podem apresentar diferença na variabilidade quando submetidas a diferentes práticas de manejo. E áreas pedologicamente diferentes, quando submetidas ao mesmo manejo, podem apresentar atributos semelhantes (CORÁ et al., 2004).

Dados georreferenciados em estudos da variabilidade espacial de atributos do solo de uma propriedade agrícola permitem a obtenção de mapas de fertilidade de solos e o estabelecimento de estratégias de aplicação de fertilizantes, sendo a análise exploratória dos dados uma etapa de fundamental importância e obrigatória em qualquer tipo de estudo deste tipo (BURROUGH et al., 1996).

A variabilidade espacial dos índices de fertilidade do solo aumenta com a adoção do sistema plantio direto, quer no sentido horizontal, pela distribuição irregular na superfície do solo (KLEPKER \& ANGHINONI, 1995; COUTO, 1997), quer ainda no sentido vertical, pelas diferenças nos teores de uma camada mais superficial em relação à outra mais abaixo (ELTZ et al., 1989; AMARAL \& ANGHINONI, 2001), demandando a definição de novos procedimentos de amostragem para contemplar essas alterações. Neste sistema, os resíduos dos vegetais provocam alterações nas propriedades físicas, químicas e biológicas do solo, causando alterações no movimento e na redistribuição de compostos mais solúveis. As freqüentes adubações e calagens em superfície tendem a formar um gradiente de concentração superficial (ELTZ et al., 1989), causando variabilidade dos índices de $\mathrm{pH}$ do solo e dos teores de nutrientes.

Além disso, independentemente do sistema de cultivo, há um limiar estreito entre toxidez e deficiência para os micronutrientes, e uma análise detalhada da área otimizaria o processo de fertilização. A matéria orgânica do solo também tem sido amplamente citada como um indicador-chave da qualidade do solo em áreas agrícolas (ZECH et al., 1997), pois exerce um papel fundamental no solo, agindo em sua estrutura pelo fornecimento de substâncias agregantes, no suprimento de macro e micronutrientes, na capacidade catiônica e tamponamento do pH (SILVA \& PASCAL, 1999). Diante disso, o objetivo deste trabalho foi avaliar a distribuição e a dependência espacial do $\mathrm{pH}$ em água, teores de matéria orgânica e micronutrientes $(\mathrm{B}, \mathrm{Cu}, \mathrm{Fe}, \mathrm{Mn}$ e $\mathrm{Zn}$ ), em três profundidades de coleta de amostras de solo, num Latossolo Vermelho distrófico cultivado há 8 anos sob sistema de semeadura direta.

\section{MATERIAL E MÉTODOS}

A área de estudo situa-se na Fazenda Santa Rosa, localizada no município de Uberlândia (MG), entre as coordenadas geográficas $18^{\circ} 55^{\prime}$ de latitude sul e $48^{\circ} 16^{\prime}$ de longitude oeste, com altitude média de $860 \mathrm{~m}$. O relevo é predominantemente suave ondulado, com declividade média de 3 a $6 \%$. O clima da região, segundo a classificação de Köppen, é Cwa, com estação seca bem definida de maio a setembro e pluviosidade média anual de $1.500 \mathrm{~mm}$. O solo da área foi classificado como Latossolo Vermelho distrófico típico textura muito argilosa, com $680 \mathrm{~g} \mathrm{~kg}^{-1}$ de argila.

O sítio de amostragem está sob cultivo de culturas anuais há mais de 20 anos, e há oito anos sob sistema de semeadura direta. A rotação de culturas na área, durante os cinco primeiros anos neste sistema, foi milho na resteva de soja a partir da safra 1995/96. Após a colheita do milho safrinha, foram utilizados, milheto, nabo forrageiro ou aveia branca como cultura de cobertura de solo, cujas sementes sempre foram semeadas a lanço.

Na implantação do sistema semeadura direta, no ano de 1995, foram aplicados e incorporados gesso agrícola e calcário (visando a elevar a saturação por bases para $65 \%$ ). A aplicação de calcário, nas safras seguintes, quando necessária, foi realizada a lanço na superfície, sem incorporação. No período de 1996 a 2000, a adubação de semeadura para a cultura da safra de verão (soja ou milho) foi realizada no sulco de semeadura, na base de $140 \mathrm{~kg} \mathrm{ha}^{-1} \mathrm{de} \mathrm{N}, 100 \mathrm{~kg} \mathrm{ha}^{-1} \mathrm{de}$ $\mathrm{P}_{2} \mathrm{O}_{5}, 90 \mathrm{~kg} \mathrm{ha}^{-1}$ de $\mathrm{K}_{2} \mathrm{O}$ e $2 \mathrm{~kg} \mathrm{ha}^{-1}$ de $\mathrm{Zn}$. Quando a cultura de verão era a soja, a adubação nitrogenada era suprimida. As culturas de cobertura de solo, milheto, nabo forrageiro ou aveia branca não receberam adubação. Na safra 2000/2001, a cultura de verão foi a soja, com aplicação de $400 \mathrm{~kg} \mathrm{ha}^{-1}$ do formulado 02-20$18+1 \mathrm{~kg} \mathrm{ha}^{-1}$ de B no sulco de semeadura, Co e Mo no tratamento de sementes e Mn via foliar; na safra 2001/ 2002, a cultura de verão foi o milho, com aplicação de 
$500 \mathrm{~kg} \mathrm{ha}^{-1}$ do formulado $08-28-18+6 \mathrm{~kg} \mathrm{ha}^{-1} \mathrm{de} \mathrm{Zn}$ na semeadura e $120 \mathrm{ha}^{-1}$ de uréia em cobertura (lanço) e, na safra 2002/2003, a cultura de verão foi o algodão, com aplicação de $650 \mathrm{~kg} \mathrm{ha}^{-1}$ do formulado $08-28-16+1 \mathrm{~kg}$ $\mathrm{ha}^{-1}$ de $\mathrm{Zn}$ no sulco de semeadura.

Em 2003, após a colheita do algodão, uma parcela de $500 \mathrm{~m} \times 500 \mathrm{~m}$ foi dividida numa malha com espaçamento regular de $50 \mathrm{~m}$ entre os pontos, resultando um quadrado de 11 linhas e 11 colunas, totalizando 121 pontos. Todos os pontos amostrados (uma amostra simples por ponto) foram georreferenciados. As amostras foram retiradas com pá de corte, transversalmente aos sulcos de semeadura e no espaço compreendido entre os pontos médios entre os sulcos, e estratificadas em três profundidades, sendo $0-10 \mathrm{~cm}, 10-20 \mathrm{~cm}$, e $0-20 \mathrm{~cm}$.

As amostras de solo, após serem secadas ao ar, peneiradas com malha de $2 \mathrm{~mm}$ de abertura, foram analisadas quimicamente, determinando-se $\mathrm{pH}$ em água na relação 1:2,5 (solo-água), B (água quente), $\mathrm{Fe}, \mathrm{Cu}$, $\mathrm{Mn}$ e $\mathrm{Zn}$ disponíveis (Mehlich-1), conforme procedimentos apresentados por SILVA(1999) e matéria orgânica, conforme RAIJ \& QUAGGIO (1983).

Os resultados encontrados foram primeiramente submetidos à análise estatística descritiva, obtendo-se média, mediana, valor máximo, valor mínimo, coeficiente de variação e coeficiente de curtose. A hipótese da normalidade dos dados foi testada pelo teste de Kolmogorov-Smirnov a 5\% de probabilidade. $\mathrm{O}$ teste $\mathrm{t}$ Student, também a $5 \%$ de probabilidade, foi aplicado para comparação das médias dos atributos entre as profundidades estudadas.

A dependência espacial dos atributos estudados foi avaliada por meio da geoestatística (VIEIRA et al., 1983), através do cálculo da semivariância e do ajuste dos dados ao semivariograma experimental. Os semivariogramas são representações gráficas entre a semivariância $\tilde{a}(\mathrm{~h})$ representada na coordenada y, em função de uma determinada distância $h$, representada na coordenada $\mathrm{x}$. A semivariância pode ser estimada pela seguinte equação:

$$
\gamma(\mathrm{h})=\frac{1}{2 \mathrm{~N}(\mathrm{~h})} \sum_{\mathrm{i}=1}^{\mathrm{N}(\mathrm{h})}\left[\mathrm{Z}\left(\mathrm{x}_{\mathrm{i}}\right)-\mathrm{Z}\left(\mathrm{x}_{\mathrm{i}}+\mathrm{h}\right)\right]^{2}
$$

em que: $N(h)$ representa o número de pares de valores medidos, $\mathrm{Z}\left(\mathrm{x}_{\mathrm{i}}\right)$ e $\mathrm{Z}\left(\mathrm{x}_{\mathrm{i}}+\mathrm{h}\right)$, separados por um vetor $(\mathrm{h}) . \mathrm{O}$ gráfico de $\tilde{a}(\mathrm{~h})$ versus os valores correspondentes de $\mathrm{h}$ é denominado semivariograma.

Os semivariogramas foram ajustados com o software Gs+ (GAMMADESIGN SOFTWARE, 2000). A seleção dos modelos foi realizada com base no melhor coeficiente de determinação $\left(\mathrm{R}^{2}\right)$ e na menor soma de quadrados do resíduo (SQR), sendo testados os modelos esférico, exponencial, gaussiano, linear, linear sem patamar e efeito pepita puro. Nos semivariogramas ajustados, foram definidos os seguintes parâmetros: efeito pepita $\left(\mathrm{C}_{0}\right)$; patamar $\left(\mathrm{C}_{0}+\mathrm{C}_{1}\right)$ e alcance da dependência espacial (a). A razão entre o efeito pepita e o patamar $\mathrm{C}_{0} /\left(\mathrm{C}_{0}+\mathrm{C}_{1}\right)$ permite a classificação e a comparação entre atributos do solo. Para analisar o grau de dependência espacial dos atributos em estudo, utilizou-se a classificação proposta por CAMBARDELLA et al. (1994), em que os semivariogramas com dependência espacial forte possuem razão menor ou igual a $25 \%$, os semivariogramas com dependência espacial moderada possuem razão superior a $25 \%$ e inferior a $75 \%$ e os de dependência fraca possuem razão superior a $75 \%$.

\section{RESULTADOS E DISCUSSÃO}

Observou-se diferença significativa apenas nos teores médios de matéria orgânica, Fe, Mn e Zn entre as profundidades estudadas (Tabela 1). Maiores teores de matéria orgânica, $\mathrm{Mn}$ e $\mathrm{Zn}$ foram encontrados nos primeiros $10 \mathrm{~cm}$ do solo, ao contrário do $\mathrm{Fe}$.

Segundo a classificação proposta por ALVAREZ et al. (1999), apenas os valores de $\mathrm{pH}$ em água e teores de $\mathrm{Zn}$ ficaram acima do nível crítico na profundidade de $0-10 \mathrm{~cm}$ e nas profundidades de 10 $20 \mathrm{~cm}$ e $0-20 \mathrm{~cm}$; além destas duas variáveis, os teores de Fe disponível também ficaram acima do nível crítico. Os teores de B e Mn (10-20 e 0-20cm) são classificados como baixos e muito baixos, respectivamente, o que sugere que as práticas de fertilização com estes micronutrientes, apesar de pontualmente apresentarem valores elevados (valores máximos, Tabela 1), não foram suficientes. Ressalta-se que os teores de $\mathrm{Zn}$ foram classificados como altos, revelando que a adubação com micronutrientes está desequilibrada, uma vez que se aplicou muito $\mathrm{Zn}$ no solo, pouco B e o $\mathrm{Mn}$ foi aplicado apenas via foliar e apenas no cultivo da soja. Além disso, a calagem pode ser responsável pelos menores teores de Mn disponível, uma vez que, em condições normais, o fator mais importante que controla a disponibilidade do Mn para as plantas é a reação do solo, decrescendo com a elevação do $\mathrm{pH}$. BORKET (1991) afirma que a deficiência de Mn ocorre quando os valores de $\mathrm{pH}$ em água são superiores a 6,2, sendo os valores de $\mathrm{pH}$ nas três profundidades superiores a este valor (Tabela 1).

Os valores de $\mathrm{pH}$ em água se apresentam homogêneos nas profundidades estudadas (Tabela 1), como resultado da incorporação do calcário na adoção do sistema de semeadura direta e, segundo CAIRES et al. (2000), pela movimentação de $\mathrm{Ca}\left(\mathrm{HCO}_{3}\right)_{2} \mathrm{e}$ $\mathrm{Mg}\left(\mathrm{HCO}_{3}\right)_{2}$ para camadas subsuperficiais, contribuindo 
Variabilidade espacial do $\mathrm{pH}$, teores de matéria orgânica e micronutrientes em profundidades de amostragem...

Tabela 1 - Valores médios, medianos, máximos, coeficiente de variação (C.V.), assimetria e curtose dos dados de $\mathrm{pH}\left(\mathrm{H}_{2} \mathrm{O}\right)$, $\mathrm{M}_{\text {.O }} \mathrm{O}$ (dag kg ${ }^{-1}$ ), B, Cu, Fe, Mn, Zn $\left(\mathrm{mg} \mathrm{dm}^{-3}\right)$, nas profundidades de 0-10, 10-20 e 0-20 cm. Fazenda Santa Rosa, Uberlândia-MG, 2004.

\begin{tabular}{|c|c|c|c|c|c|c|c|c|}
\hline Variável & Média ${ }^{(1)}$ & Mediana & $\mathrm{d}^{(2)}$ & Assimetria & Curtose & Mínimo & Máximo & $\mathrm{CV}(\%)$ \\
\hline \multicolumn{9}{|c|}{$0-10 \mathrm{~cm}$} \\
\hline $\mathrm{pH} \mathrm{em} \mathrm{H}_{2} \mathrm{O}$ & $6,47^{\mathrm{ns}}$ & 6,50 & $0,05^{\mathrm{ns}}$ & $-0,22$ & $-0,74$ & 5,60 & 7,10 & 5,83 \\
\hline M.O. & $3,67^{*}$ & 3,70 & $0,04^{\mathrm{ns}}$ & $-0,08$ & $-0,03$ & 2,90 & 4,50 & 9,28 \\
\hline B & $0,35^{\mathrm{ns}}$ & 0,35 & $0,07^{\mathrm{ns}}$ & 0,50 & 0,07 & 0,11 & 0,83 & 40,56 \\
\hline $\mathrm{Cu}$ & $0,86^{\mathrm{ns}}$ & 0,80 & $0,06^{\mathrm{ns}}$ & 0,59 & $-0,09$ & 0,40 & 1,60 & 27,14 \\
\hline $\mathrm{Fe}$ & $39,6^{*}$ & 39,70 & $0,04^{\mathrm{ns}}$ & 0,40 & $-0,43$ & 19,00 & 60,00 & 24,82 \\
\hline Mn & $3,32^{*}$ & 3,20 & $0,06^{\mathrm{ns}}$ & 0,41 & $-0,21$ & 1,90 & 5,50 & 22,06 \\
\hline $\mathrm{Zn}$ & $3,46^{*}$ & 3,30 & $0,05^{\mathrm{ns}}$ & 0,33 & 0,33 & 1,20 & 5,50 & 21,57 \\
\hline \multicolumn{9}{|c|}{$10-20 \mathrm{~cm}$} \\
\hline $\mathrm{pH} \mathrm{em} \mathrm{H}_{2} \mathrm{O}$ & 6,26 & 6,25 & $0,04^{\mathrm{ns}}$ & 0,08 & $-0,98$ & 5,52 & 7,00 & 6,05 \\
\hline M.O. & 3,38 & 3,30 & $0,03^{\text {ns }}$ & 0,29 & $-0,10$ & 2,40 & 4,20 & 10,29 \\
\hline B & 0,31 & 0,33 & $0,07^{\mathrm{ns}}$ & 0,62 & 0,83 & 0,10 & 0,77 & 42,07 \\
\hline $\mathrm{Cu}$ & 0,80 & 0,80 & $0,05^{\mathrm{ns}}$ & 0,63 & 0,48 & 0,30 & 1,90 & 32,13 \\
\hline $\mathrm{Fe}$ & 46,7 & 46,00 & $0,06^{\mathrm{ns}}$ & 0,32 & $-0,04$ & 24,00 & 60,00 & 22,95 \\
\hline $\mathrm{Mn}$ & 1,88 & 1,80 & $0,04^{\mathrm{ns}}$ & 0,52 & 0,91 & 1,80 & 5,10 & 36,16 \\
\hline $\mathrm{Zn}$ & 2,66 & 2,50 & $0,05^{\mathrm{ns}}$ & 0,24 & $-0,98$ & 1,80 & 5,30 & 23,37 \\
\hline \multicolumn{9}{|c|}{$0-20 \mathrm{~cm}$} \\
\hline $\mathrm{pH} \mathrm{em} \mathrm{H}_{2} \mathrm{O}$ & 6,30 & 6,30 & $0,04^{\mathrm{ns}}$ & 0,16 & $-0,42$ & 5,50 & 7,00 & 5,56 \\
\hline M.O. & 3,58 & 3,60 & $0,05^{\mathrm{ns}}$ & $-0,02$ & $-0,13$ & 2,70 & 4,20 & 8,21 \\
\hline B & 0,33 & 0,35 & $0,07^{\mathrm{ns}}$ & 0,36 & $-0,36$ & 0,10 & 0,71 & 39,13 \\
\hline $\mathrm{Cu}$ & 0,82 & 0,80 & $0,06^{\mathrm{ns}}$ & 0,63 & 0,74 & 0,30 & 1,60 & 30,52 \\
\hline $\mathrm{Fe}$ & 44,1 & 43,50 & $0,06^{\mathrm{ns}}$ & 0,33 & $-0,60$ & 26,00 & 70,00 & 23,54 \\
\hline Mn & 2,80 & 2,70 & $0,05^{\mathrm{ns}}$ & 0,65 & 0,06 & 1,50 & 5,30 & 29,94 \\
\hline $\mathrm{Zn}$ & 3,01 & 2,85 & $0,07^{\mathrm{ns}}$ & 0,47 & $-0,18$ & 2,10 & 5,20 & 23,67 \\
\hline
\end{tabular}

${ }^{(1)}$ Média de 121 amostras $={ }^{n s} \mathrm{e}^{*}$, significam diferença não-significativa e diferença significativa a 5\% de probabilidade pelo teste t Student, respectivamente.

(2) $\mathrm{d}=$ estatística do teste Kolmogorov-Smirnov ; ${ }^{\mathrm{ns}}$ não significativo a 5\% pelo referido teste.

para o aumento do $\mathrm{pH}$ nestas camadas. Esta movimentação é favorecida neste sistema, uma vez que possui características físicas ideais ao movimento. Ocorre também a formação de complexos de compostos orgânicos solúveis lixiviados da palhada superficial e da matéria orgânica com Ca e Mg (MIYASAWA et al., 1993) e Al trocável (PAVAN, 1997). Na camada subsuperficial, o Ca e o $\mathrm{Mg}$ dos compostos são deslocados pelo Al do solo, formando complexos mais estáveis, diminuindo a acidez trocável e aumentando os teores de cátions básicos nesta camada.

Em geral, os teores de Mn e Zn decresceram em profundidade, sendo os menores teores verificados na camada de $10-20 \mathrm{~cm}$, o que se atribui aos menores teores de matéria orgânica nessa camada (Tabela 1). Esses resultados concordam com os obtidos por CASTRO et al. (1992) e TEIXEIRA et al. (2003), no que se refere aos teores disponíveis destes micronutrientes (extrator DTPA), que foram maiores na semeadura direta, em comparação com o sistema convencional, tendo as diferenças sido notadas na camada superficial do solo. No entanto, esses autores encontraram diferença significativa nos teores de $\mathrm{Cu}$, nas diferentes profundidades, no sistema semeadura direta, o que não foi verificado neste trabalho. Segundo HOROWITZ \& DANTAS (1976), ao longo do perfil, a adsorção do Zn é função do teor e qualidade da argila. Os autores também citam que o Zn permanece vários anos na superfície e que o movimento lateral é bastante reduzido. Os maiores teores de $\mathrm{Mn}$ e $\mathrm{Zn}$ nos primeiros $10 \mathrm{~cm}$ de solo podem ser explicados pela maior quantidade de matéria orgânica na referida camada, uma vez que a matéria orgânica é uma das principais fontes destes nutrientes no solo, e pela adubação ter sido realizada na superfície do solo.

Dados os valores elevados de $\mathrm{pH}$ e os teores de matéria orgânica nos primeiros $10 \mathrm{~cm}$, os teores de $\mathrm{Fe}$ foram menores nesta camada que nas outras profundidades avaliadas (Tabela 1). TEIXEIRA et al. (2003) encontraram correlação linear negativa apenas 
para $\mathrm{Fe}$, entre os teores de carbono orgânico e teores dos micronutrientes catiônicos no sistema de semeadura direta, devido ao poder complexante da matéria orgânica do solo. RHOTON (2000) relata que a maior quantidade de matéria orgânica do solo no sistema de semeadura direta reduziu a disponibilidade de Fe pela formação de complexos Fe-ácido fúlvico. Ademais, de acordo com SHUMAN \& HARGROVE (1985), não ocorre aumento nos teores de Fe nos primeiros centímetros do solo, apesar de ele ser reciclado em maior proporção que $\mathrm{Zn}$ e Mn, devido à sua rápida oxidação ao ser liberado da matéria orgânica, o que justificaria esses resultados.

A análise descritiva dos dados, apesar de considerar os dados espacialmente independentes, tem a função de analisar o comportamento geral dos dados e identificar algum dado que possa exercer qualquer influência na análise geoestatística. Os resultados desta análise (Tabela 1) indicaram distribuição normal. Os valores de média e mediana estão próximos em todas as variáveis, mostrando distribuições quase simétricas. Outro indicativo da normalidade são os valores de coeficientes de assimetria e curtose próximos de zero (Tabela 1). O teste de normalidade KolmogorovSmirnov confirmou a distribuição normal de todos os dados (Tabela 1).

Os valores de assimetria e de curtose para a distribuição normal devem ser de 0 e 3 , respectivamente (SNEDECOR \& COCHRAN, 1967); porém, o programa GS+ utiliza o valor de 0 para estes dois parâmetros. A maioria das variáveis apresentou coeficientes de assimetria positivos, sendo os maiores valores para manganês $(0-20 \mathrm{~cm})$, de 0,65 , e o menor para $\mathrm{pH}$ (10$20 \mathrm{~cm})$, de 0,08 . Coeficientes de assimetria negativos também foram verificados, sendo maior para $\mathrm{pH}$ em água $(0-10 \mathrm{~cm})$, de - 0,22, e o menor para $\mathrm{MO}(0-20 \mathrm{~cm})$, de $-0,02$. Foi verificado menor número de coeficientes de assimetria negativos (3). O coeficiente de assimetria é utilizado para caracterizar como e quanto a distribuição de freqüências se afasta da simetria. Se o valor encontrado para este coeficiente for zero, a distribuição é simétrica; se for positivo, a distribuição é assimétrica à direita e, se for negativo, é assimétrica à esquerda.

Os valores máximos e mínimos encontrados nas três profundidades foram semelhantes (Tabela 1). Quando se analisam as amplitudes entre os valores mínimos e máximos, pode-se ter uma noção da variabilidade dos dados. SOUZA (1992) observou correlação diretamente proporcional entre esta amplitude e os respectivos coeficientes de variação (CV). No presente trabalho, os menores coeficientes de variação foram observados para pH em água e os maiores para B (Tabela 1). A amplitude dos dados representou bem seus respectivos coeficientes de variação. Por exemplo, o valor máximo do $\mathrm{pH}$ em água encontrado na profundidade de $0-20 \mathrm{~cm}$ (menor CV) foi de 1,27 vezes maior que o valor mínimo. Para $B$, por sua vez, na profundidade de $10-20 \mathrm{~cm}$ (maior CV), esta diferença foi de 7,7 vezes.

Com base no critério de WARRICK \& NIELSEN (1980), as variáveis $\mathrm{pH}$ em água e matéria orgânica apresentaram $\mathrm{CVs}$ baixos $(\mathrm{CV}<12 \%)$ e $\mathrm{B}, \mathrm{Cu}$, Fe, Mn e Zn CVs médios (12,1\% < CV <60\%). Estas classificações se repetiram nas três profundidades estudadas. Em vários trabalhos também foram encontrados $\mathrm{CVs}$ baixos para $\mathrm{pH}$ em água e $\mathrm{MO}$ (SOUZA, 1992; SANTOS \& VASCONCELLOS, 1987; SCHLINDWEIN \& ANGHINONI, 2000). São poucos os dados na literatura sobre variabilidade de micronutrientes em sistema de semeadura direta. SILVA et al. (2000) também encontraram CVs médios para os micronutrientes catiônicos, com exceção do $\mathrm{Zn}(\mathrm{CV}>$ $70 \%$ ), além de SILVA et al. (2002). Os CVs médios podem ser atribuídos ao efeito residual da adubação e ao modo de aplicação contínua de fertilizantes, mecanicamente e na linha de semeadura. Maiores variações para os teores de micronutrientes podem ser explicados pelo manejo da adubação, pela amostragem e pela característica de cada nutriente avaliado. Menores CVs do pH em água, por exemplo, podem ser explicados pela baixa concentração hidrogeniônica na solução do solo da área e da matéria orgânica, pela sua distribuição mais uniforme na área.

A análise geoestatística, efetuada por meio dos semivariogramas, mostrou que todas as variáveis apresentaram dependência espacial, exceto $\mathrm{Zn}$ na profundidade de $0-20 \mathrm{~cm}$ (Tabela 2). Quando a variável estudada é independente espacialmente, o seu semivariograma se ajusta ao modelo linear, em que $\mathrm{C} 0$ é igual a $\mathrm{C}_{1}+\mathrm{C}_{0}$, conhecido como efeito pepita puro (EPP). O EPP é importante e indica distribuição casual, e pode ser devido a erros de medidas ou microvariação nãodetectada, considerando ser o espaçamento de amostragem utilizado maior que o necessário para detectar dependência espacial (CAMBARDELLA et al., 1994).

Um parâmetro importante no estudo dos semivariogramas é o alcance, que significa a distância máxima que uma variável está correlacionada espacialmente (DAVIS, 1986), ou seja, determinações realizadas a distâncias maiores que o alcance têm distribuição aleatória e, por isso, são independentes entre si. O alcance é uma medida importante para planejamento e avaliação experimental, podendo auxiliar na definição de procedimentos amostrais (WEBSTER, 1985), tendo aplicação direta no plano de amostragem na agricultura de precisão.

O menor valor de alcance foi de $27 \mathrm{~m}$ para $\mathrm{B}$ 
Tabela 2 - Estimativas dos parâmetros dos modelos dos semivariogramas ajustados para as variáveis pH $\left(\mathrm{H}_{2} \mathrm{O}\right), \mathrm{M}^{\mathrm{O}} \mathrm{O}\left(\mathrm{dag} \mathrm{kg}^{-1}\right), \mathrm{B}, \mathrm{Cu}, \mathrm{Fe}$, $\mathrm{Mn}, \mathrm{Zn}\left(\mathrm{mg} \mathrm{dm}^{-3}\right)$, nas profundidades de 0-10, 10-20 e 0-20 cm. Fazenda Santa Rosa, Uberlândia-MG, 2004.

\begin{tabular}{|c|c|c|c|c|c|c|}
\hline Variável & $\mathrm{C}_{0}{ }^{(1)}$ & $\mathrm{C}_{1}+\mathrm{C}_{0}{ }^{(2)}$ & Alcance (m) & Modelo $^{(3)}$ & $\begin{array}{c}\text { Dependência (\%) } \\
{\left[\mathrm{C}_{0} / \mathrm{C}_{0}+\mathrm{C}_{1}\right] \times 100}\end{array}$ & Classificação \\
\hline \multicolumn{7}{|c|}{$0-10 \mathrm{~cm}$} \\
\hline $\mathrm{pH}$ & 0,022 & 0,145 & 352 & ESF & 15,17 & Forte \\
\hline M.O. & 0,032 & 0,117 & 283 & EXP & 27,35 & Moderada \\
\hline B & 0,016 & 0,047 & 58 & LIN & 34,04 & Moderada \\
\hline $\mathrm{Cu}$ & 0,028 & 0,055 & 152 & EXP & 50,91 & Moderada \\
\hline $\mathrm{Fe}$ & 30,40 & 103,0 & 77 & EXP & 29,51 & Moderada \\
\hline $\mathrm{Mn}$ & 0,114 & 0,434 & 63 & EXP & 26,27 & Moderada \\
\hline $\mathrm{Zn}$ & 0,184 & 0,694 & 57 & EXP & 26,51 & Moderada \\
\hline \multicolumn{7}{|c|}{$10-20 \mathrm{~cm}$} \\
\hline $\mathrm{pH}$ & 0,0235 & 0,0473 & 310 & LIN & 49,68 & Moderada \\
\hline M.O. & 0,0402 & 0,3020 & 250 & LIN & 13,31 & Forte \\
\hline B & 0,0049 & 0,0108 & 27 & EXP & 45,37 & Moderada \\
\hline $\mathrm{Cu}$ & 0,0177 & 0,0679 & 59 & ESF & 26,07 & Moderada \\
\hline $\mathrm{Fe}$ & 14,700 & 72,390 & 60 & EXP & 20,31 & Forte \\
\hline Mn & 0,1300 & 0,4840 & 48 & ESF & 26,86 & Moderada \\
\hline $\mathrm{Zn}$ & 0,3320 & 1,3490 & 37 & EXP & 24,61 & Forte \\
\hline \multicolumn{7}{|c|}{$0-20 \mathrm{~cm}$} \\
\hline $\mathrm{pH}$ & 0,0240 & 0,0990 & 250 & ESF & 24,24 & Forte \\
\hline M.O. & 0,0589 & 0,1390 & 259 & LIN & 42,37 & Moderada \\
\hline B & 0,0044 & 0,0209 & 49 & ESF & 21,05 & Forte \\
\hline $\mathrm{Cu}$ & 0,0276 & 0,0553 & 152 & EXP & 49,91 & Moderada \\
\hline $\mathrm{Fe}$ & 21,400 & 103,40 & 29 & EXP & 20,70 & Forte \\
\hline Mn & 0,2203 & 0,4416 & 46 & EXP & 49,89 & Moderada \\
\hline $\mathrm{Zn}$ & 0,0453 & 0,0453 & - & EPP & - & - \\
\hline
\end{tabular}

(1) $\mathrm{C}_{0}$ representa o efeito pepita; ${ }^{(2)} \mathrm{C}_{1}+\mathrm{C}_{0}$ representa o patamar; ${ }^{(3)}$ Modelo: ESF. = esférico; EXP $=$ exponencial; LIN $=$ linear; EPP $=$ efeito pepita puro.

$(10-20 \mathrm{~cm})$ e o maior, de $352 \mathrm{~m}$, para $\mathrm{pH}(0-10 \mathrm{~cm})$ (Tabela 2). Observando-se os valores de alcance encontrados para as três profundidades, verifica-se que estas características são diferentes. Nota-se que se for adotada a geoestatística no esquema de amostragem, o número de amostras a serem coletadas nas profundidades de 0-10, 10-20 e 0-20cm será distinto, possivelmente coletando-se um número menor de amostras na primeira, devido aos maiores valores do alcance apresentados por todas as variáveis nesta profundidade. Portanto, além da variabilidade vertical, há também variabilidade horizontal entre as profundidades, visto que o alcance foi diferente para algumas variáveis, nas diferentes profundidades.

Ajustou-se o modelo exponencial para matéria orgânica $(0-10 \mathrm{~cm})$, boro $(10-20 \mathrm{~cm})$, cobre $(0-10$ e 10-20), ferro (nas três profundidades), manganês (010 e $0-20 \mathrm{~cm})$ e zinco $(0-10$ e $10-20 \mathrm{~cm})$. O modelo esférico foi ajustado para $\mathrm{pH}$ em água $(0-10$ e $0-20 \mathrm{~cm})$, boro (0$20 \mathrm{~cm})$, cobre $(10-20 \mathrm{~cm})$ e manganês $(10-20 \mathrm{~cm})$. Para matéria orgânica (10-20 e 0-20cm) e boro $(0-10 \mathrm{~cm})$, foi ajustado o modelo linear (Tabela 2 ).
Para analisar o grau de dependência espacial dos atributos em estudo, utilizou-se a classificação proposta por CAMBARDELLA et al. (1994). A análise do efeito pepita dos atributos químicos do solo revelou que, nas profundidades de $0-10$ e $10-20 \mathrm{~cm}$, houve a predominância de dependência espacial moderada e, na profundidade de $0-20 \mathrm{~cm}$, houve empate entre a quantidade de dependência espacial forte e dependência moderada (Tabela 2). Isso demonstra que os semivariogramas explicam a maior parte da variância dos dados.

Os resultados obtidos devem ser considerados na definição de planos de amostragem do solo e no manejo da adubação da área em questão através da análise do alcance e da variabilidade horizontal e vertical dos atributos químicos estudados.

\section{CONCLUSÕES}

Maiores teores de matéria orgânica, Mn e $\mathrm{Zn}$ foram verificados nos primeiros $10 \mathrm{~cm}$ do solo, 
enquanto que os maiores teores de Fe ocorreram na camada de $10-20 \mathrm{~cm}$. O coeficiente de variação indicou baixa variabilidade para $\mathrm{pH}$ em água e matéria orgânica e média para os micronutrientes em todas as profundidades avaliadas, com todas as variáveis seguindo distribuição normal. Com exceção do Zn (0$20 \mathrm{~cm}$ ), os demais atributos estudados apresentaram dependência espacial, classificada como moderada ou forte, com alcances variando de 27 a $352 \mathrm{~m}$. A maioria dos dados se ajustou ao semivariograma de modelo exponencial, seguido do esférico e do linear.

\section{REFERÊNCIAS}

ALVAREZ V. et al. Interpretação dos resultados das análises de solos. In: RIBEIRO, A.C. et al. Recomendações para o uso de corretivos e fertilizantes em Minas Gerais. 5. Aproximação. Viçosa: CFSEMG, 1999. p.25-32.

AMARAL, A.S.; ANGHINONI, I. Alterações de parâmetros químicos do solo pela reaplicação superficial de calcário no sistema plantio direto. Pesquisa Agropecuária Brasileira, Brasília, v.36, n.4, p.695-702, 2001.

BORKET, C.M. Manganês. In: FERREIRA, M.E; CRUZ, M.C.P. Micronutrientes na agricultura. Piracicaba: Potafos, 1991. p.173-88.

BURROUGH, P.A. et al. Using public domain geostatistical and GIS software for spatial interpolation. Photogrammetric Engineering \& Remote Sensing, Falls Church, v.62, n.7, p.845-854, 1996.

CAMBARDELLA, C.A. et al. Field-scale variability of soil properties in central Iowa soils. Soil Science Society of America Journal, Madison, v.58, n.5, p.1501-1511, 1994.

CAIRES, E.F. et al. Calagem na superfície em sistema plantio direto. Revista Brasileira de Ciência do Solo, Viçosa, v.24, n.1, p.161-169, 2000.

CASTRO, O.M. et al. Teores de zinco, cobre, manganês e ferro em dois Latossolos sob plantio direto e convencional. Bragantia, Campinas, v.51, n.1, p.77-84, 1992.

CORÁ, J.E. et al. Variabilidade espacial de atributos do solo para adoção do sistema de agricultura de precisão na cultura de cana-de-açúcar. Revista Brasileira de Ciência do Solo, Viçosa, v.28, n.6, p.1013-1021, 2004.

COUTO, E.G. Variabilidade espacial de propriedades do solo influenciado pela agricultura em escala regional e local no sul do estado do Mato Grosso. 1997. 183f. Tese (Doutorado em Agronomia) - Curso de Pós-graduação em Ciência do Solo, Universidade Federal do Rio Grande do Sul.

DAVIS, J.C. Statistics and data analysis in geology. 2.ed. New York: Jonh Wiley, 1986. 646p.

DEUTSCH, C.V.; JOURNEL, A.G. GSLIB: geostatistical software library and user's guide, New York: Oxford University, 1998. 369p. 1 CD-ROM.
ELTZ, F.L.P. et al. Efeitos de sistemas de preparo do solo nas Propriedades físicas e químicas de um latossolo brunoálico. Revista Brasileira de Ciência do Solo, Campinas, v.13, p.259-267, 1989.

GAMMA DESIGN SOFTWARE. Geoestatistics for the environmental sciences (version 5.1 for windows). Michigan, 2000. 1 CD ROM.

HOROWITZ, A.; DANTAS, H.S. Geoquímica dos elementos menores nos solos de Pernambuco. IV. Zinco na zona LitoralMata. Pesquisa Agropecuária Brasileira: Série Agronômica, Rio de Janeiro, v.11, n.12, p.27-35, 1976.

KLEPKER, D.; ANGHINONI, I. Características físicas e químicas do solo afetadas por métodos de preparo e modos de adubação. Revista Brasileira Ciência do Solo, Campinas, v.19, n.3, p.395-401, 1995.

MIYAZAWA, M. et al. Efeito de material vegetal na acidez do solo. Revista Brasileira de Ciência do Solo, Campinas, v.17, n.3, p.411-416, 1993.

PAVAN, M.A. Ciclagem de nutrientes e mobilidade de íons no solo sob plantio direto. Revista Plantio Direto, Passo Fundo, v.41, p.8-12, 1997.

RAIJ, B. van; QUAGGIO, J.A. Métodos de análise de solo para fins de fertilidade. Campinas: Instituto Agronômico de Campinas, 1983. 40p. (Boletim Técnico, 81).

RHOTON, F.E. Influence of time on soil response to no-till practices. Soil Science Society of America Journal, Madison, v.64, n.2, p.700-709, 2000.

SANTOS, H.L.; VASCONCELLOS, C.A. Determinação do número de amostras de solo para análise química em diferentes condições de manejo. Revista Brasileira de Ciência do Solo, Campinas, v.11, n.2, p.97-100, 1987.

SCHLINDWEIN, J.A., ANGHINONI, I. Variabilidade horizontal de atributos de fertilidade e amostragem do solo no sistema plantio direto. Revista Brasileira de Ciência do Solo, Viçosa, v.24, n.1, p.85-91, 2000.

SHUMAN, L.M.; HARGROVE, L. Effect of tillage on the distribution of manganese, copper, iron and zinc in soil fractions. Soil Science Society of America Journal, Madison, v.49, n.5, p.1117-1121, 1985 .

SILVA, C.A. et al. Amostragem e variabilidade de atributos de solo e planta em área de soja sob plantio direto. In: FERTIBIO, 2000, Santa Maria, RS. Anais... Santa Maria: Universidade Federal de Santa, Universidade Federal de Santa Maria, SBCS, SBM, 2000. 4p. (CD ROM).

SILVA, F.C. Manual de análises químicas de solos, plantas e fertilizantes. Embrapa Solos, Embrapa Informática Agropecuária. Brasília: Embrapa Comunicação para transferência de tecnologia, 1999. 370p.

SILVA, L.M.V.; PASQUAL, A. Dinâmica e modelagem da matéria orgânica do solo com ênfase ao ecossistema tropical. Energia na Agricultura, Botucatu, v.14, n.3, p.13-24, 1999. 
SILVA, M.A.G. et al. Amostragem e variabilidade dos atributos do solo em área de soja sob plantio direto. Acta Scientiarum Maringá, v.24, n.5, p.1197-1204, 2002.

SNEDECOR, G.W.; COCHRAN, W.G. Statistical methods. 6.ed. Ames, Iowa: Iowa State University, 1967. 593p.

SOUZA, L.S. Variabilidade espacial do solo em sistemas de manejo. 1992. 162f. Tese (Doutorado em Agronomia) Curso de Pós-graduação em Ciência do Solo, Universidade Federal do Rio Grande do Sul.

TEIXEIRA, I.R. et al. Variação dos valores de $\mathrm{pH}$ e dos teores de carbono orgânico, cobre, manganês, zinco e ferro em profundidade em Argissolo Vermelho-Amarelo, sob diferentes sistemas de preparo de solo. Bragantia, Piracicaba, v.62, p.119-126, 2003
VIEIRA, S.R. et al. Geostatitical theory and application to variability of some agronomical properties. Hilgardia, Berkeley, v.51, n.3, p.1-75, 1983.

WARRICK, A.W.; NIELSEN, D.R. Spatial variability of soil physical properties in the field. In: HILLEL, D. (Ed). Applications of soil physics. New York: Academic, 1980. Cap.2, p.319-44.

WEBSTER, R. Quantitative spatial analysis of soil in the field. In: STEWART, B.A. (Ed). Advance in soil science. New York: Springer-Verlag, 1985. V.3, p.1-70.

ZECH, Z. et al. Factors controlling humification and mineralization of soil organic matter in the tropics. Geoderma, Amsterdam, v.79, n.1, p.69-116, 1997. 